\title{
A RAS recruitment screen identifies ZKSCAN4 as a glucocorticoid receptor-interacting protein
}

\author{
Karin Ecker ${ }^{1}$, Andreas Lorenz ${ }^{1}$, Frank Wolf ${ }^{1}$, Christian Ploner ${ }^{1}$, Günther Böck ${ }^{2}$, Tod Duncan ${ }^{3}$, \\ Stephan Geley ${ }^{1}$ and Arno Helmberg ${ }^{1}$
}

\author{
${ }^{1}$ Biocenter, Division of Molecular Pathophysiology, Innsbruck Medical University, Fritz Pregl Strasse 3, A 6020 Innsbruck, Austria \\ ${ }^{2}$ Biocenter, Division of Developmental Immunology, Innsbruck Medical University, Fritz Pregl Strasse 3, A 6020 Innsbruck, Austria \\ ${ }^{3}$ Department of Biology, University of Colorado, Denver, 1200 Larimer Street, NC 3016 E, Denver, Colorado 80204, USA \\ (Correspondence should be addressed to A Helmberg; Email: arno.helmberg@i-med.ac.at)
}

\begin{abstract}
To search for proteins interacting with the glucocorticoid receptor, we adapted Aronheim's reverse RAS recruitment system relying on the Saccharomyces cerevisiae mutant cdc25-2, which has a temperature-dependent defect in its RAS signaling pathway driving proliferation. The full-length human glucocorticoid receptor (NR3C1, isoform- $\alpha$ ) was attached to the yeast plasma membrane in either of two orientations and used as bait to screen a HeLa cell cDNA library. Library proteins were fused to constitutively active, soluble human RAS, complementing the defective yeast pathway in case of bait-prey interaction. Screening of 800000 clones resulted in the isolation of 21 proteins, 8 of which were followed up to evaluate interaction with the receptor in human cell lines. One of these candidates, the SCAN- and KRAB-domaincontaining zinc finger protein 307 (ZKSCAN4) was co-precipitated with the receptor when both proteins were overexpressed in HEK293 cells. Rabbit antisera against ZKSCAN4 were raised, affinity purified, and used to immunoprecipitate endogenous ZKSCAN4 from Hct116 cells, resulting in co-precipitation of endogenous glucocorticoid receptor. Overexpressed ZKSCAN4 was found to co-localize in granular nuclear structures with the activated glucocorticoid receptor and partially with chromatin regions characterized by histone $\mathrm{H} 3$ mono-methylated on lysine 4 (H3K4me1). Overexpressed ZKSCAN4 had no effect on an episomal glucocorticoid receptor-driven reporter plasmid. By contrast, ZKSCAN4 markedly reduced glucocorticoid induction of the mouse mammary tumor virus-promoter-driven reporter gene when this was chromosomally integrated, arguing for a chromatin-dependent inhibition of glucocorticoid receptor-mediated transactivation.
\end{abstract}

Journal of Molecular Endocrinology (2009) 42, 105-117

\section{Introduction}

Glucocorticoids, a class of steroid hormones released from the adrenal gland, play a key role in the regulation of metabolism, immune responses, and inflammatory reactions (Sapolsky et al. 2000, Necela \& Cidlowski 2004). Synthetic equivalents are widely used as drugs to treat allergic, autoimmune, and certain malignant diseases (Schimmer \& Parker 2005). Glucocorticoids enter the cell by free diffusion across the lipid bilayer of the cell membrane. In the cytoplasm, they interact with the glucocorticoid receptor (GR), which, in the absence of ligand, is bound to a large, dynamic multiprotein chaperone complex. This complex includes heat shock protein molecules Hsp90 and, temporarily, Hsp70 as well as an immunophilin component and is essential to keep the receptor in a high affinity state for hormone binding (Dittmar et al. 1997, Pratt \& Toft 1997). Upon association with its ligand, the ubiquitously expressed receptor translocates to the nucleus where it acts as a transcriptional regulator, interacting either directly with DNA or with other proteins involved in transcriptional regulation.

Interactions with other proteins have been extensively investigated in two functional settings: first, for the cytoplasmic multi-protein complex containing the unliganded receptor (Pratt et al. 2006) and secondly, for the liganded, nuclear receptor in the context of transcriptional regulation (Necela \& Cidlowski 2004, Copik et al. 2006). Much less effort has been devoted to investigating potential effects of the GR without pertinence to transcription, meaning important interactors might have been missed so far (Stellato 2004). Sengupta et al. described ligand-dependent interaction of the GR with p53 and MDM2, building up a trimeric complex in the cytoplasm. Interaction of these proteins leads to cytoplasmic sequestration and proteasome-dependent degradation of both GR and TP53. (Sengupta et al. 2000, Sengupta \& Wasylyk 2001). In one recent systematic study, the GR was immunoprecipitated from cytosolic rat 
liver extracts, and co-immunoprecipitated proteins were separated on 2D-gels, resulting in hundreds of spots, of which 34 were identified by mass spectrometry (Hedman et al. 2006). However, these candidates were not followed up. To also allow the detection of cytoplasmic interaction partners, we used the GR as a cytoplasmic, membranebound bait to perform an unbiased search for further interacting proteins.

A typical tool to search for protein-protein interactions is the yeast two-hybrid (Y2H) system. In its classical form, the system makes use of the reconstitution of transcription factor activity via the interaction of two proteins (the 'prey' and the 'bait'), one fused to the activation domain of a transcription factor and the other fused to the corresponding DNA-binding domain, to facilitate expression of reporter genes (Fields \& Song 1989). This system has the inherent limitation that proteins containing transactivating domains cannot be used as bait, as they would directly activate the expression of reporter genes. Consequently, only parts of the GR excluding the large transactivation domains have so far been used in Y2H-screens (Gottlicher et al. 1996, Hittelman et al. 1999, Yang et al. 2000). To overcome this limitation, Aronheim and co-workers developed alternative cytoplasmic systems, one of which, the reverse RAS recruitment system (rRRS), we adapted, using the GR as bait (Aronheim 2001, Hubsman et al. 2001); instead of a transcription factor activity, the temperature-dependently defective RAS-signaling pathway is reconstituted in the Saccharomyces cerevisiae mutant strain $c d c 25-2$. This strain (Petitjean et al. 1990) harbors a temperature-sensitive mutation (E1328K) in CDC25, rendering it inactive and unable to activate RAS at the restrictive temperature of $37^{\circ} \mathrm{C}$. The $\mathrm{GR} \alpha$-isoform was used as bait, attached to the yeast plasma membrane by an N-terminal myristoylation or a C-terminal farnesylation sequence. Prey proteins expressed from a HeLa cDNA library were fused to constitutively active human (h) RAS lacking its farnesylation domain $(\operatorname{RAS}(61) \Delta \mathrm{F}$; Broder et al. 1998). The RAS mutant is able to substitute for the defective yeast protein if brought into the proximity of the membrane via the interaction of the cDNA-encoded moiety with the GR. Thus, growth signaling at the restrictive temperature is reconstituted, resulting in colony formation. In contrast to other systems, this procedure allows the identification of proteins interacting with the full-length GR under cytoplasmic conditions of a eukaryotic cell and results in the isolation of a defined span of cDNA as a starting point for follow-up experiments.

\section{Materials and methods}

\section{Expression plasmids for the $\mathrm{Y} 2 \mathrm{H}$ system}

GR-expression plasmids pKECKhGR-myr and pKECKhGR-CAAX are based on the vector pADNS
(LEU2; ampR) (Colicelli et al. 1989). A multiple cloning site containing a HindIII as well as a NotI restriction site was inserted and the ADH1-promoter was exchanged by the Met25-promoter derived from plasmid pMet25 (Mumberg et al. 1994) using standard cloning techniques. The ampicillin resistance gene was replaced by a kanamycin resistance gene by recombination in strain EL250 (Lee et al. 2001) as described (Yu et al. 2000), forming the plasmid pKECK. Of the different human splice variants and isoforms (Yudt \& Cidlowski 2001, Presul et al. 2007), the classical GR $\alpha$ (A) open reading frame (ORF) was amplified by standard PCR technique from plasmid pEF $\Delta$ T-hGRwt (Riml et al. 2004) in frame with either a C-terminal CAAX-sequence (leading to post-translational farnesylation) encoded in the reverse primer or with an N-terminal myristoylation (Myr)sequence encoded in the forward primer and subsequently ligated into the HindIII/NotI restricted vector PKECK.

The yeast RAS-fusion cDNA library (URA3; ampR) from proliferating HeLa cells was generated as previously described (Broder et al. 1998). Fusion proteins were expressed under control of the GAL1promoter. Constitutively active, soluble hRAS (RAS (61) $\Delta \mathrm{F}$ ) is fused N-terminally to the protein fragments encoded by oligo-dT-primed HeLa cDNA. The library had a complexity of 800.000 clones before a single round of amplification.

\section{Expression plasmids for human cell lines}

cDNAs for candidate genes were obtained from RZPD (clone IRAUp696B0365D for ZKSCAN4). Coding sequences for full-length ZKSCAN4, the different truncated mutants, and GR $\alpha$ were amplified by standard PCR technology and cloned into plasmid pDONR207 (Invitrogen), followed by further subcloning into Flagor cyan fluorescent protein (CFP)-destination vectors using GATEWAY technology (Invitrogen, Carlsbad, CA, USA), resulting in plasmids pE-FLAG-ZKSCAN4 or pE-CFP-ZKSCAN4 respectively. The GR was expressed without tag (pE-GR) or, for the purpose of microscopy studies, in frame with a yellow fluorescent variant (YFP)tag at the N-terminus (pE-YFP-GR). Plasmid phPR-SG5, based on vector pSG5 (Stratagene, La Jolla, CA, USA), contains the full-length cDNA of the human progesterone receptor (PR) under control of the SV40promoter and was a kind gift of Dr Wolfgang Doppler, Biocenter, Innsbruck Medical University.

\section{Plasmids for bacterial overexpression}

To obtain a 6xHis-tagged version of ZKSCAN4, the coding sequence was subcloned into vector pDEST17 (Invitrogen) using GATEWAY technology (pE-6xHis). 
For the purpose of antibody affinity purification, an N-terminal fragment of the ORF (ZKSCAN4 (AA 1-194)) was subcloned into a destination vector encoding N-terminal GST (pE-GST-ZKSCAN4).

\section{Yeast media, growth conditions and transfections}

$S$. cerevisiae strain $c d c 25-2$ has been previously described (Petitjean et al. 1990). Yeast cells were grown at 25 or $37^{\circ} \mathrm{C}$ on glucose or galactose minimal medium containing $0 \cdot 17 \%$ Difco yeast nitrogen base without amino acids, $0.5 \% \mathrm{NH}_{4} \mathrm{SO}_{4}$, the appropriate added amino acids and $2 \%$ glucose or a mixture of $3 \%$ galactose, $2 \%$ raffinose, and $1 \%$ glycerol. For plates, $4 \%$ of agar was added. Methionine was supplemented at a concentration of $200 \mu \mathrm{g} / \mathrm{ml}$ to repress methionine-promoter-dependent transcription. Transfections were performed as previously described (Aronheim et al. 1994).

\section{Cell culture, transfections, and lysis conditions}

U2OS, Hct116, and HEK-293T cells were maintained in DMEM (Cambrex, East Rutherford, NJ, USA), CEMC7H2 and Jurkat cells were cultured in RPMI medium (Cambrex), both supplemented with 10\% FCS (PAA laboratories $\mathrm{GmbH}$, Linz, Austria), $100 \mathrm{U} / \mathrm{ml}$ penicillin, $100 \mu \mathrm{g} / \mathrm{ml}$ streptomycin, and $0.3 \mathrm{mg} / \mathrm{ml}$ glutamine (Cambrex). DNA and siRNA transient transfections were performed using Lipofectamine 2000 reagent (Invitrogen) according to the manufacturer's protocol. For inducing the GR for nuclear translocation, dexamethasone (dex) was added to the medium at a final concentration of $10^{-7} \mathrm{M}$. For total lysates, cells were washed with PBS, harvested from the culture dish by a rubber policeman, centrifuged at 1000 r.p.m. for $5 \mathrm{~min}$, and lysed in NP-40 based lysis buffer $(20 \mathrm{mM}$ Tris-HCl (pH 8), $137 \mathrm{mM} \mathrm{NaCl}, 10 \%$ glycerol, $1 \% \mathrm{NP}-$ 40, $2 \mathrm{mM}$ EDTA) for $1 \mathrm{~h}$ at $4{ }^{\circ} \mathrm{C}$. CEM-C7H2 cells were stably transfected with a ZKSCAN4-expression construct by retroviral transfection.

\section{Generation of Jurkat GR MMTV-VNP cells}

The lentiviral pHR-MMTV-VNP construct was generated by inserting the mouse mammary tumor virus LTRpromoter (MMTV) from pMMTV-Luc (Doppler et al. 2001) into the MluI-SnabI sites of pHR-MCS-VNP. The latter plasmid was constructed by PCR amplifying venus-NLS-Pest (VNP (Nagoshi et al. 2004)) using primers $5^{\prime}$-tatagaattcggatcccacgcgtgtttaaactacgtagccaccatggtgagcaagggc- $3^{\prime}$ (sense) and $5^{\prime}$-tatagcggccgcccatatgactagttacacattgatcc- $3^{\prime}$ (antisense). The purified fragment, containing a primer-generated multiple cloning site and VNP, was inserted into the EcoRI/NotI-sites of the lentiviral plasmid pHR-SIN-CSGW- $\Delta$ Not (Demaison et al. 2002), thereby replacing the spleen focus-forming virus (SFFV)-eGFP cassette, to generate pHR-MCS-VNP.

\section{Generation of MMTV-VNP expressing cells}

Preparation of lentiviral supernatant was described elsewhere (Ploner et al. 2008). In brief, $3 \mu \mathrm{g}$ of the lentiviral construct together with $2 \mu \mathrm{g}$ pCMV 8.91 and $2 \mu \mathrm{g}$ pMD-G plasmids were transfected into the HEK293T packaging cell line. Lentiviral supernatant was harvested 48 and $72 \mathrm{~h}$ after transfection, sterile filtered, supplemented with $4 \mu \mathrm{g} / \mu \mathrm{l}$ polybrene (Sigma St Louis, MO, USA) and $5 \times 10^{5}$ Jurkat-GR cells (Helmberg et al. 1995) were infected twice with $500 \mu$ l lentiviral supernatant.

\section{Generation of ZKSCAN4 overexpressing Jurkat GR MMTV-VNP cells}

The lentiviral constitutive expression plasmid pHRSFFV-ZKSCAN4-ires-Puro was generated by recombining pENTR-307-ZKSCAN4 with the 'destination vector' pHR-SFFV-Dest-ires-Puro (Ploner et al. in preparation), facilitating the simultaneous expression of ZKSCAN4 and, for selection, the puromycin resistance gene under control of the SFFV-promoter. The bulk of MMTV-VNP expressing Jurkat GR cells were subsequently infected with the lentiviral construct coding for ZKSCAN4, following the same procedure as described above.

\section{Co-immunoprecipitation experiments}

For co-immunoprecipitation (co-IP) experiments with overexpressed proteins, $24 \mu \mathrm{g}$ DNA were used to transfect HEK-293T and Hct116 cells in a $10 \mathrm{~cm}$ dish at $90 \%$ confluency with plasmids pE-FLAG-ZKSCAN4 and pE-GR. $48 \mathrm{~h}$ after transfection, cells were lysed in a HEPES-based lysis buffer $(0 \cdot 2 \%$ Triton X-100; $50 \mathrm{mM}$ HEPES ( $\mathrm{pH} 7 \cdot 5$ ); $150 \mathrm{mM} \mathrm{NaCl} ; 10 \%$ glycerol; protease inhibitor cocktail from Roche; $50 \mathrm{mM} \mathrm{NaF} ; 5 \mathrm{mM}$ $\mathrm{NaOV}$ ) for $1 \mathrm{~h}$ at $4{ }^{\circ} \mathrm{C}$ followed by incubation of $1 \mathrm{mg}$ lysate with $10 \mu \mathrm{g}$ FLAG-antibody (F3165; SigmaAldrich), $5 \mu \mathrm{g}$ ZKSCAN4-antibody, or $5 \mu \mathrm{g}$ GR-antibody (sc-1003; Santa-Cruz Biotechnology, Santa Cruz, CA, USA) respectively. After incubation for $1 \mathrm{~h}$ at $4{ }^{\circ} \mathrm{C}$, lysates were centrifuged for $15 \mathrm{~min}$ at 13000 r.p.m. to eliminate potential antibody-antigen super-complexes. Supernatant was further incubated with protein G-agarose beads (E3403; Sigma-Aldrich) for $1 \mathrm{~h}$ at $4{ }^{\circ} \mathrm{C}$ followed by four washing steps with the lysis buffer. The co-IP samples were subjected to SDS-PAGE and western blotting with FLAG- or GR-antibody respectively. As a negative control, lysates were incubated with 
protein G-Sepharose beads only or with isotype controls.

\section{ZKSCAN4-antibody production and affinity purification}

A 6xHis-tagged version of ZKSCAN4 was expressed in E.coli strain BL21 (DE3)pLysS from plasmid pE-6xHisZKSCAN4 upon induction with $2 \mathrm{mM}$ IPTG and purified by binding to Ni-NTA agarose (1018244; Qiagen). Purified fusion protein together with Freund's adjuvant was used to immunize two rabbits in 4-week intervals over a total time period of 6 months. The first immunization was performed with complete (F-5991; Sigma), further immunizations with incomplete Freund's adjuvant (F-5506; Sigma). To affinity purify the antibody, a GST-tagged version of an N-terminal fragment of the protein (GST-ZKSCAN4 ${ }_{(\mathrm{AA} 1-194)}$ ) was bound to a Glutathione-Sepharose matrix (17-0756-01; Amersham) and covalently cross-linked using $40 \mathrm{mM}$ dimethyl pimelinediimidate (80490; Fluka) in $0 \cdot 1 \mathrm{M}$ borate buffer ( $\mathrm{pH} 9)$. After application of rabbit antiserum to the column, bound ZKSCAN4-antibody was eluted with $0 \cdot 2 \mathrm{M}$ glycine $(\mathrm{pH} \mathrm{2 \cdot 0)}$ ) and buffered immediately. The resulting affinity-purified antibodyeluate was unstable and only usable for about $10 \mathrm{~d}$ at $4{ }^{\circ} \mathrm{C}$, and was therefore frozen in small aliquots upon purification.

\section{Fluorescence microscopy and immunostaining}

Microscopy was performed with an Axiovert 200M live cell imaging instrument and a Zeiss Axiovert 100 confocal microscope with LSM510 laserhead. For live cell microscopy, home-made glass-bottomed culture dishes were used and cells were monitored at $37^{\circ} \mathrm{C}$ and $5 \% \mathrm{CO}_{2}$.

For immunostaining experiments, cells were fixated with methanol for $4 \mathrm{~min}$ at $-20^{\circ} \mathrm{C}$. Antibodies for immunostaining: Y12 (Acris; DM3198); mono-methyl H3K4 (Abcam; ab8895); tri-methyl H3K4 (Abcam; ab8580); PML (Santa Cruz Biotechnology; Sc-966); our self-made affinity purified rabbit anti-ZKSCAN4; Alexa Fluor anti-rabbit 488 (Invitrogen; A11008); Alexa Fluor anti-rabbit 568 (Invitrogen; A10042); Alexa Fluor antimouse 594 (Invitrogen; A11062).

\section{Site-directed mutagenesis}

To mutate the two arginines in the sequence of CFP-

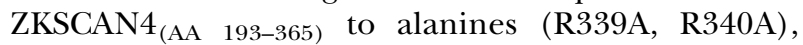
site-directed mutagenesis was performed by PCR with Pfu-polymerase using forward and reverse primers containing the appropriate nucleotides under standard PCR conditions. DpnI restriction was performed to disrupt the old methylated DNA template. Competent E. coli cells were transformed with the PCR product and isolated plasmids (CFP-ZKSCAN4 ${ }_{(\mathrm{AA}}$ 193-365) $\left.\Delta \mathrm{NLS}\right)$ were checked by sequencing.

\section{Immunoblotting}

Lysates were boiled in Laemmli buffer $(250 \mathrm{mM}$ Tris$\mathrm{HCl}$, (pH 6.8); $20 \%$ glycerol; $6 \%$ SDS; $0.1 \%$ bromophenol blue; $1,43 \mathrm{M} \beta$-mercaptoethanol) for $5 \mathrm{~min}$, separated by SDS-PAGE on a $12.5 \%$ gel and electrophoretically transferred onto a nitrocellulose membrane. Membranes were blocked with $5 \%$ defatted milk in TBS-T for $1 \mathrm{~h}$ at $4{ }^{\circ} \mathrm{C}$ followed by incubation with the primary antibody, diluted in TBS-T, over night at $4{ }^{\circ} \mathrm{C}$. After three washing steps, blots were incubated with secondary antibodies coupled to horse radish peroxidase and specific bands were detected using Immobilon HRP detection reagent (Millipore, Bedford, MA, USA). Antibodies used for immunoblotting: FLAG (F3165; Sigma-Aldrich, St Louis, MO, USA); GR (sc-1003; Santa-Cruz Biotechnology); affinity purified rabbit anti-ZKSCAN4; GAPDH (ab9484; Abcam, Cambridge, UK).

\section{Results}

\section{Screening for GR-interacting proteins by the reverse RAS recruitment system}

Two different yeast $c d c 25-2$ strains were generated by transfection with either GR-expression vector pKECKhGR-myr or pKECK-hGR-CAAX. In these cells, the full-length GR $\alpha$ (bait) was expressed as fusion protein with an $\mathrm{N}$-terminal myristoylation or a C-terminal farnesylation (CAAX) sequence. Membrane anchorage of the receptor by these post-translational lipid modifications was verified by western blot analysis of membrane and soluble fractions of cell lysates. The GR was expressed under control of the methioninerepressible Met25-promoter. High methionine concentrations strongly reduced the expression of the bait, while lack of methionine in the medium allowed expression, as shown in Fig. 1A for the GR-CAAX fusion protein.

A flow diagram of the screening procedure is shown in Fig. 1B. Strain $c d c 25-2$ expressing either one of the GR fusions was transfected with the Gall-promoterdriven RAS-fusion HeLa cDNA library. After incubation on glucose minimal medium at the permissive temperature of $25^{\circ} \mathrm{C}$, library colonies were replica plated onto two galactose-containing plates differing in methionine concentrations $(0 \mu \mathrm{g} / \mathrm{ml}$ or $200 \mu \mathrm{g} / \mathrm{ml})$ and incubated at the restrictive temperature of $37^{\circ} \mathrm{C}$. Down-regulation of GR-expression by methionine allowed testing for 


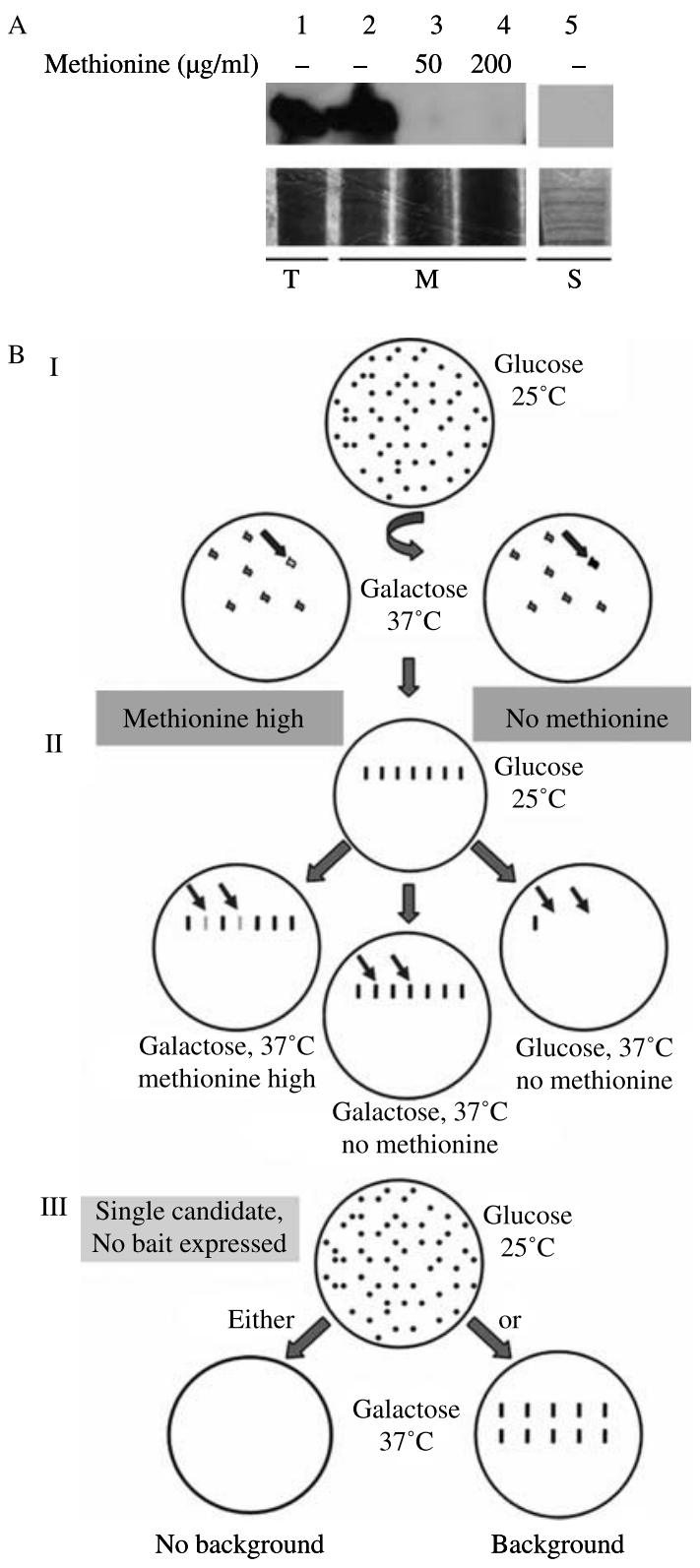

Figure 1 Screening procedure. (A) Expression of GR-CAAX in cdc25-2. GR-expression was repressed by an addition of methionine to the growth medium (lanes 3 and 4). A high level of protein was found in total cell lysates (lane $1 ; T$ ) and in the membrane fraction of cell lysates (lane 2; M). No signal could be detected in the soluble fraction (lane 5; S). Lower panel, Coomassie staining. (B) Flowchart. Step I: after 3 days of growth on glucose medium at $25^{\circ} \mathrm{C}$, colonies were replica plated onto galactose plates. Colonies that showed preferred growth on the plate lacking methionine after 3 days of incubation at $37^{\circ} \mathrm{C}$ (indicated by an arrow) were picked, transferred to glucose plates, cultivated at $25^{\circ} \mathrm{C}$, and tested again for methionine-dependent difference in growth ability on galactose $/ 37^{\circ} \mathrm{C}$ in a first verification step (positive clones: arrows in step II). Step III: Positive clones were transfected into strain cdc25-2 lacking bait. If this clone (10 independent colonies) was unable to grow at the restrictive temperature, the cDNA insert was sequenced. false positive library proteins that attach to the membrane per se. Replica plating on a glucose plate allowed identification of potential revertants. After incubation at the restrictive temperature, colonies showing preferred growth on the plate lacking methionine were picked as candidates and subjected to a reconfirmation step. In a last verification procedure, the corresponding prey plasmid was isolated and transfected into $c d c 25-2$ devoid of the GR-expression construct to once more exclude potential bait-independent growth. Screening was performed in the presence and absence of $10^{-6} \mathrm{M}$ deoxycorticosterone, a glucocorticoid that, in contrast to dex, has been shown to be active in yeast (Garabedian \& Yamamoto 1992), but no candidates were observed that unequivocally mediated growth only in the presence of hormone.

Of 800.000 colonies screened, 70 primary candidates were finally isolated and sequenced. Sequencing allowed to eliminate $70 \%$ of these candidates from further consideration. Some of the eliminated candidates showed fusions of the cDNA to RAS in another than the ORF, frequently resulting in an artificial short, hydrophobic protein fragment. A subset of positive, but characteristically very small colonies was found to only contain the poly-A tail of cDNA, resulting in a polylysine peptide with obviously some non-specific affinity to the GR. Other candidate fragments derived from ORF within the $3^{\prime}$ untranslated regions of mRNAs. Although potentially interesting, these candidates were not further pursued, as were a few proteins with established extracellular roles. The remaining 21 candidates are listed in Table 1.

One of this small number of candidates, HSPA5, has already been described to interact with the GR in vitro (Hutchison et al. 1996), serving as a positive control, and another, TRAP1, is a close mitochondrial relative of Hsp90, the main chaperone in the cytoplasmic GR-complex. TRAP1 binding to the GR would thus not be surprising, but was not further verified.

\section{A novel binding partner for the GR: ZKSCAN4}

Based on the information available in the literature and databases, we selected eight candidate proteins for the next screening step, consisting of expressing full-length FLAG-tagged versions of the respective proteins in human cells and assaying for potential co-IP with the GR. In this step, interaction was confirmed for the $62 \mathrm{kDa}$ (545 AA) zinc finger protein 307 (ZKSCAN4; Homo sapiens zinc finger with KRAB and SCAN domains 4/ZKSCAN4). HEK-293T cells were co-transfected with expression vectors encoding GR and N-terminally FLAG-tagged zinc finger protein (FLAG-ZKSCAN4 $4_{\text {full }}$ length)). Immunoprecipitation of the receptor from total cell lysates resulted in co-precipitation of ZKSCAN4 as detected with the FLAG-antibody (Fig. 2). 
Table 1 Potential glucocorticoid receptor (GR)-interacting proteins, identified using the reverse RAS recruitment system (rRRS)

Protein
AHS1

CYPA

$\mathrm{DBI}$ (isoform 3)

YBX1

DYNLRB1

EEF1A1

EEF1G

GNL3

HSPA5

TRAP1

KRT10

MRPL4

RPL37

LonP1

MPRIP

Rho

PNAS 101

PSMB6

RAN

THMB10

ZKSCAN4

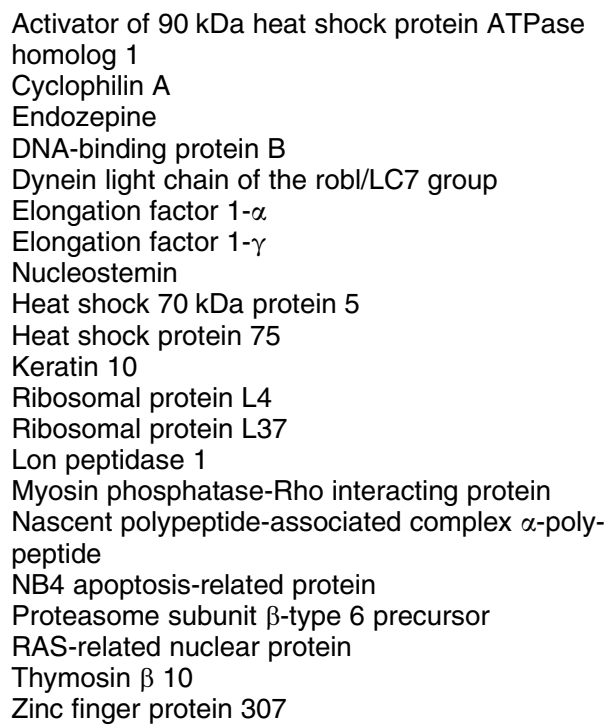

C

C

$\mathrm{C}, \mathrm{M}$

$\mathrm{C}, \mathrm{M}$

C

C

$\mathrm{NO}$

ER

$\mathrm{M}, \mathrm{C}$

$\mathrm{C}, \mathrm{N}$

C

C

$\mathrm{M}$

C

$\mathrm{C}, \mathrm{N}$

?

$\mathrm{C}$

$\mathrm{C}, \mathrm{N}$

$\mathrm{C}, \mathrm{N}$

$\mathrm{N}$

C, cytoplasm; ER, endoplasmic reticulum; M, mitochondrium; N, nucleus; NO, nucleolus.

To examine interaction of the GR with the endogenous zinc finger protein, we generated rabbit antisera against the full-length protein and subsequently affinity-purified specific antibodies using the GST-coupled N-terminal SCAN domain. Using this antibody, we tested the expression of ZKSCAN4 in five human cell lines by western blot analysis, finding higher levels of protein in HeLa, Hct116, and CEM-C7H2 (Fig. 3A; lanes 2, 4, and 6) than in U2OS and HEK-293T cells, where ZKSCAN4 was hardly detectable (not shown). As control, lysates of HeLa and Hct116 cells, transfected with plasmid pE-FLAGZKSCAN4 (lanes 1 and 3), or lysates of stably

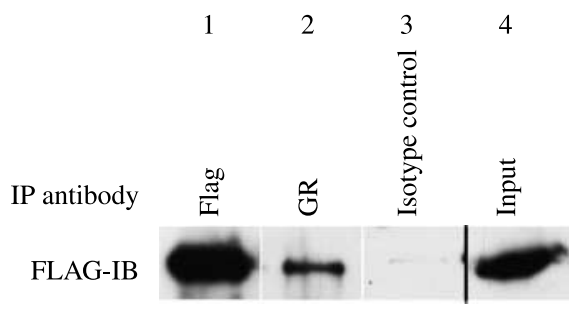

Figure 2 Overexpressed Flag-ZKSCAN4 co-immunoprecipitates with GR. HEK-293T cells were transiently transfected with expression vectors encoding FLAG-ZKSCAN4 and GR. Cells were lysed and equivalent amounts of lysates were immunoprecipitated with antibody against FLAG or GR or isotype control antibody as indicated. Immunoprecipitates were separated by SDS-PAGE and probed by immunoblotting using anti-FLAG. Input represented $4 \%$ of the lysate used in one IP reaction.
ZKSCAN4-transfected CEM-C7H2 (lane 5) cells were used. Using the affinity-purified antibody to immunostain endogenous ZKSCAN4 in fixated HeLa cells demonstrated an exclusively nuclear, granular distribution (Fig. 3B). Specificity of staining was controlled for by co-transfected siRNA: siRNA against ZKSCAN4 abrogated staining, while siRNA against unrelated luciferase did not.

Immunoprecipitation of endogenous ZKSCAN4 from Hct116 cell lysates using the affinity-purified antibody resulted in co-precipitation of the endogenous GR (Fig. 4A), confirming the interaction between the two proteins at physiological expression levels. Co-precipitation of endogenous proteins could not be demonstrated in HeLa or CEM-C7H2 cells. While ZKSCAN4 was identified as a GR-interacting protein, it conceivably might also interact with other nuclear receptors. To test for potential interaction between the PR and ZKSCAN4, we co-transfected HEK-293T cells with plasmids leading to the overexpression of these two proteins. Immunoprecipitation of ZKSCAN4 did not result in co-immunoprecipitation of PR (Fig. 4B).

As with other candidate proteins, we compared the spatial distribution of ZKSCAN4 to that of the GR by live cell wide-field and confocal fluorescence microscopy. In U2OS osteosarcoma cells, ZKSCAN4 was expressed as a hybrid protein with an N-terminal CFP. The GR was fused to the YFP. CFP-ZKSCAN4 was localized 


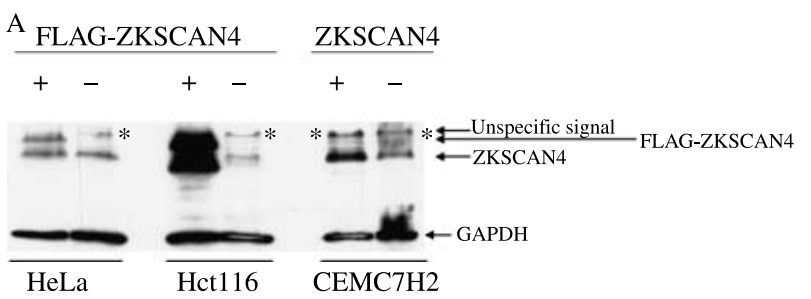

B

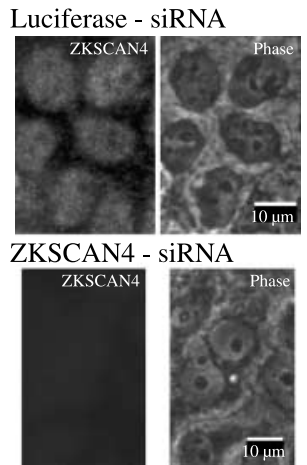

Figure 3 Characterization of an affinity-purified rabbit antibody raised against ZKSCAN4. (A) Detection of endogenous and overexpressed ZKSCAN4 in human cell line immunoblots. Whole cell lysates of HeLa, Hct116, and CEMC7H2 cells were prepared either from untransfected cells $(-)$ or from cells transiently transfected with FLAG-ZKSCAN4 or stably transfected with untagged ZKSCAN4 (CEM-C7H2) as indicated $(+)$, and sequentially probed with anti-ZKSCAN4 and anti-GAPDH. Note that in the FLAG-ZKSCAN4-transfected lanes, part of the overexpressed protein is not FLAG-tagged due to usage of the normal in-frame start codon. Slightly higher than the FLAG-ZKSCAN4, an unspecific band $\left({ }^{*}\right)$ is recognized by the ZKSCAN4-antibody. (B) Detection of endogenous ZKSCAN4 by immunocytochemistry. HeLa cells were transiently transfected with luciferase(as control) or ZKSCAN4-specific siRNA, fixated and stained with affinity purified rabbit antibody against ZKSCAN4, and fluorescence and phase contrast images were taken.

exclusively in the nucleus in either of two patterns: in a granular pattern in the nucleoplasm as seen with the endogenous protein, sometimes forming a ring-like structure around the nucleoli (Fig. 5A), or homogeneously distributed in the nucleoli (Fig. 5B). While the GR was cytoplasmic in U2OS cells washed with serumfree medium, dex-addition shifted the receptor to the nucleus within 5 min where it first showed a slightly granulated pattern followed by homogeneous nuclear distribution, as shown in a time lapse acquisition in Fig. 5C. Concomitant overexpression of CFP-ZKSCAN4 led to a redistribution of YFP-GR to the granular nucleoplasmic structures of ZKSCAN4 (confocal image, Fig. 5E). Live cell microscopy showed that the GR was recruited to the speckled CFP-ZKSCAN4 pattern immediately after nuclear translocation, until the patterns became virtually identical (Fig. 5D; note the difference in YFP-GR distribution to Fig. 5C).

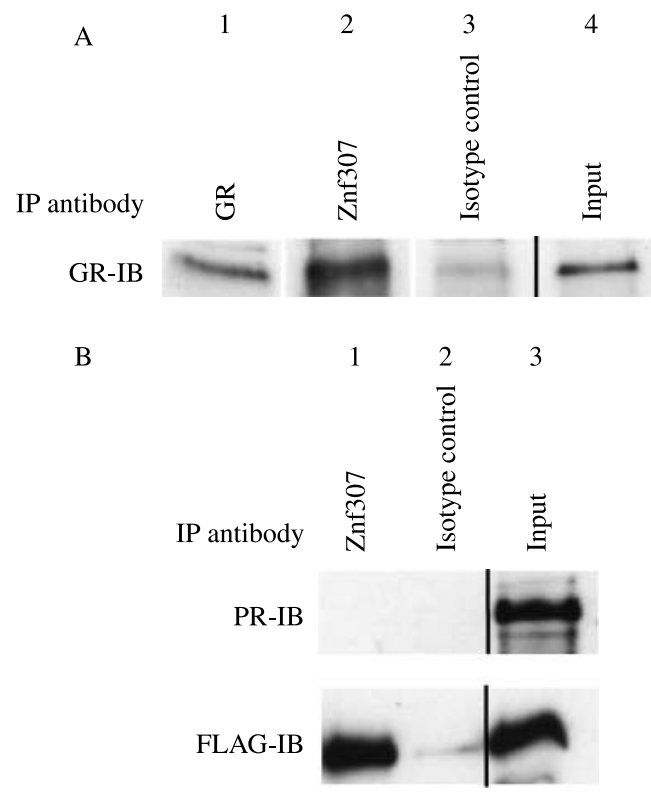

Figure 4 (A) Endogenous GR is co-immunoprecipitated with endogenous ZKSCAN4. Hct116 cells were treated with dex for $12 \mathrm{~h}$ and lysed. Equivalent amounts of lysates were immunoprecipitated with antibody against GR, ZKSCAN4, or isotype control antibody as indicated. Immunoprecipitates were separated by SDS-PAGE and probed by immunoblotting using GR-antibody. (B) ZKSCAN4 does not co-immunoprecipitate the progesterone receptor. HEK-293T cells were transiently transfected with expression vectors encoding FLAG-ZKSCAN4 and PR. Cells were lysed and equivalent amounts of lysates were immunoprecipitated with antibody against ZKSCAN4 or isotype control as indicated. Immunoprecipitates were separated by SDS-PAGE and probed by immunoblotting using anti-PR and anti-FLAG antibodies. Input represented $4 \%$ of the lysate used in one IP reaction.

\section{Identification of a ZKSCAN4 nuclear localization signal}

ZKSCAN4 contains three conserved domains: an $\mathrm{N}$-terminal SCAN, or leucine-rich domain, the namegiving zinc finger domain at the C-terminus comprising seven C2H2 zinc fingers, and a middle KRAB-(Kruppelassociated box)-A domain. To narrow down the domains of ZKSCAN4 essential for co-localization with the GR, we constructed expression vectors for each individual domain and combinations thereof (Fig. 6A), expressed in frame with an N-terminal CFP, and monitored their subcellular localization relative to the GR (Fig. 6B). The two N-terminal domain fragments CFP-ZKSCAN4 $\left.4_{(A A} 1-294\right)$ and CFP-ZKSCAN4 $\left.4_{(A A} 1-194\right)$ ) lost their ability for nuclear translocation and localized entirely in the cytoplasm. The small CFP-ZKSCAN4 ${ }_{\text {(AA }}$ 193-294) was homogeneously distributed, probably due to passive diffusion of the $41 \mathrm{kDa}$ protein through the nuclear pore complex. All the other fragments resided in the nucleus. Only CFP-ZKSCAN4 ${ }_{(\mathrm{AA}}$ 193-545), 

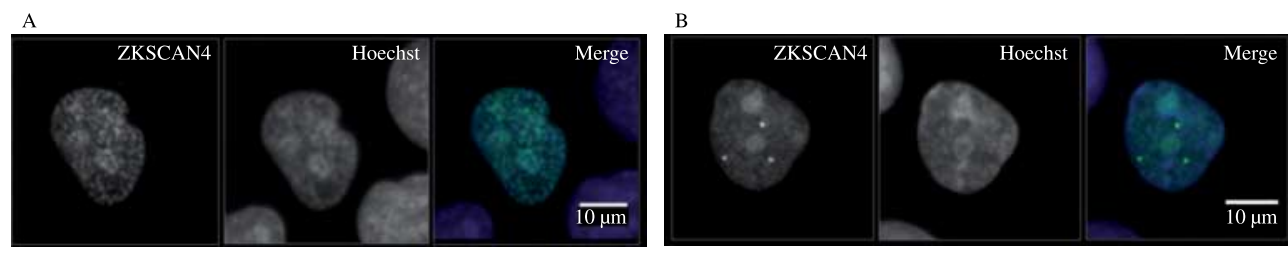

C
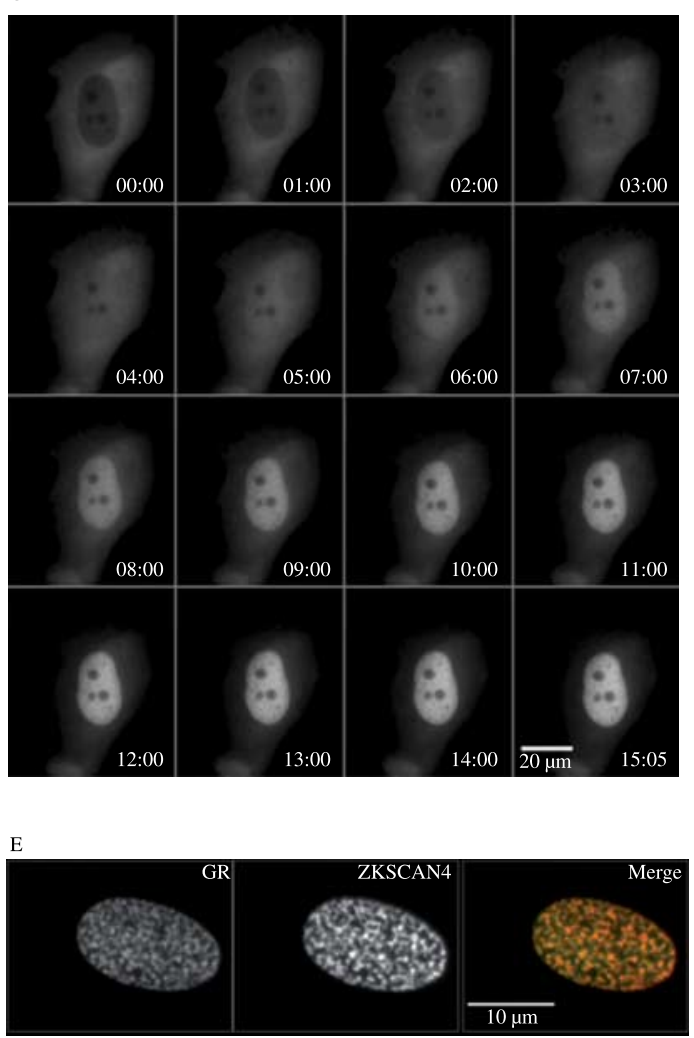

Figure 5 Subnuclear localization of ZKSCAN4 and the GR. (A, B) U2OS cells were transiently transfected with an expression vector encoding CFP-ZKSCAN4, stained with Hoechst 33342, and fluorescence images were taken as indicated. (C) U2OS cells were transiently transfected with an expression vector encoding YFP-GR, treated with $10^{-7} \mathrm{M}$ dex, and filmed. Panels show fluorescence images taken every minute. (D) U2OS cells were transiently transfected with expression vectors encoding CFP-ZKSCAN4 and YFP-GR, treated with $10^{-7} \mathrm{M}$ dex and filmed. Panels show fluorescence images taken every $10 \mathrm{~min}$. (E) U2OS cells were transiently transfected with expression vectors encoding CFP-ZKSCAN4 and YFP-GR, treated with $10^{-7} \mathrm{M}$ dex, and confocal fluorescence images were taken after $15 \mathrm{~h}$.

consisting of KRAB plus zinc finger domain, co-localized with the induced GR in the typical granular pattern as shown for the full-length protein in Fig. 5D and E. The C-terminal fragment CFP-ZKSCAN4 ${ }_{\text {(AA 366- }}$ 545) showed nucleolar localization, indicating the presence of a nucleolar targeting signal sequence in the zinc finger domain.

The observed difference in cellular location between the two fragments containing only the KRAB-domain (CFP-ZKSCAN4 ${ }_{(\mathrm{AA} 193-294)}$ and CFP-ZKSCAN4 ${ }_{(\mathrm{AA} 193-}$ 365) ) suggested the presence of a nuclear localization signal (nls) between amino acids 295 and 365 as the longer fragment was exclusively nuclear. This area contains a KHRR sequence that might function as nuclear targeting sequence. By exchanging the two arginines to alanines by site-directed mutagenesis in the $49 \mathrm{kDa}$ fusion protein CFP-ZKSCAN4 ${ }_{(\mathrm{AA}}$ 193-365) (R339A; R340A), the fragment lost its exclusively nuclear localization, proving the nls-function of amino acids KHRR (Fig. 6C). By contrast, exchange of the two amino acids in the full-length construct did not prevent nuclear localization (data not shown). Therefore, the presence of additional nuclear localization signals in the zinc finger domain seems likely. 

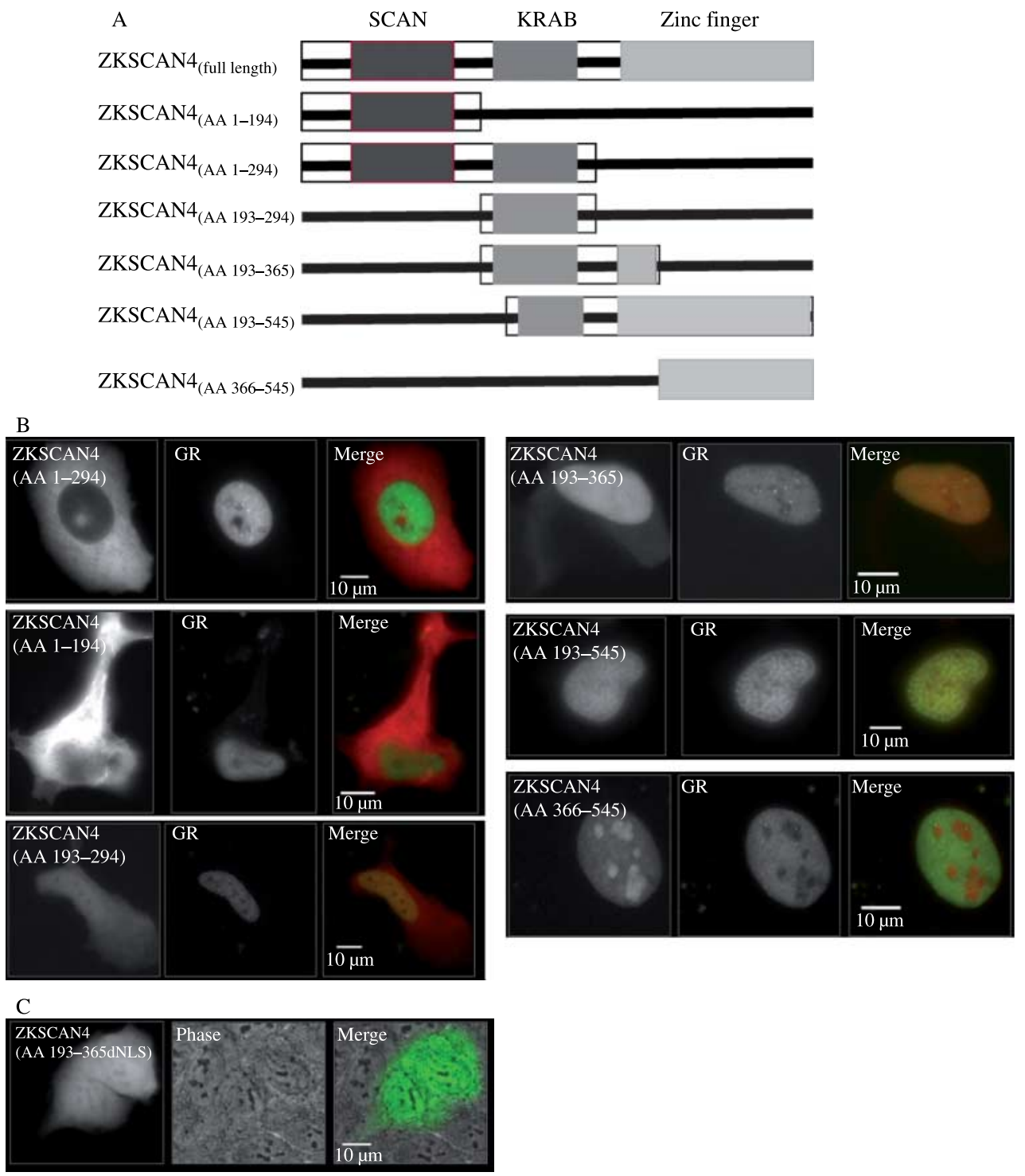

Figure 6 Cellular localization of ZKSCAN4 domain constructs and identification of a nuclear localization sequence. (A) Schematic of the ZKSCAN4-parts of CFP-ZKSCAN4 fusion proteins. (B) U2OS cells were transiently transfected with expression vectors encoding the indicated individual CFP-ZKSCAN4 domain construct and YFP-GR, treated with $10^{-7} \mathrm{M}$ dex and fluorescence images were taken after $12 \mathrm{~h}$. (C) U2OS cells were transiently transfected with an expression vector encoding CFP-ZKSCAN4 $\left.{ }_{(\mathrm{AA}} 193-365\right) \Delta \mathrm{NLS}$, and fluorescence and phase contrast images were taken after $40 \mathrm{~h}$.

\section{Relationship of ZKSCAN4 to known subnuclear structures}

To assess whether ZKSCAN4 might be part of known subnuclear structures, we compared the distribution of ZKSCAN4 with the distributions of informative proteins. No overlap was found with the Y12 protein, which is typical for the classical 'speckles' involved in splicing (Lamond \& Spector 2003). Also, there was no overlap with PML-bodies, which form a distinct speckled pattern and have been reported to be involved in transcriptional regulation (Fig. 7B; Zhong et al. 2000), or with nuclear regions enriched for histone $\mathrm{H} 3$ tri-methylated on lysine 9 , which is typical for regions that have been silenced by conversion to heterochromatin (Fig. 7C). However, we found partial co-localization of ZKSCAN4 with nuclear regions enriched in histone $\mathrm{H} 3$ mono-methylated on lysine 4 (H3K4me1) which may be poised for transcriptional activation (Schneider et al. 2004) (Fig. 7D). By contrast, very little co-localization was observed for the tri-methylated form H3K4me3 (Fig. 7E). 

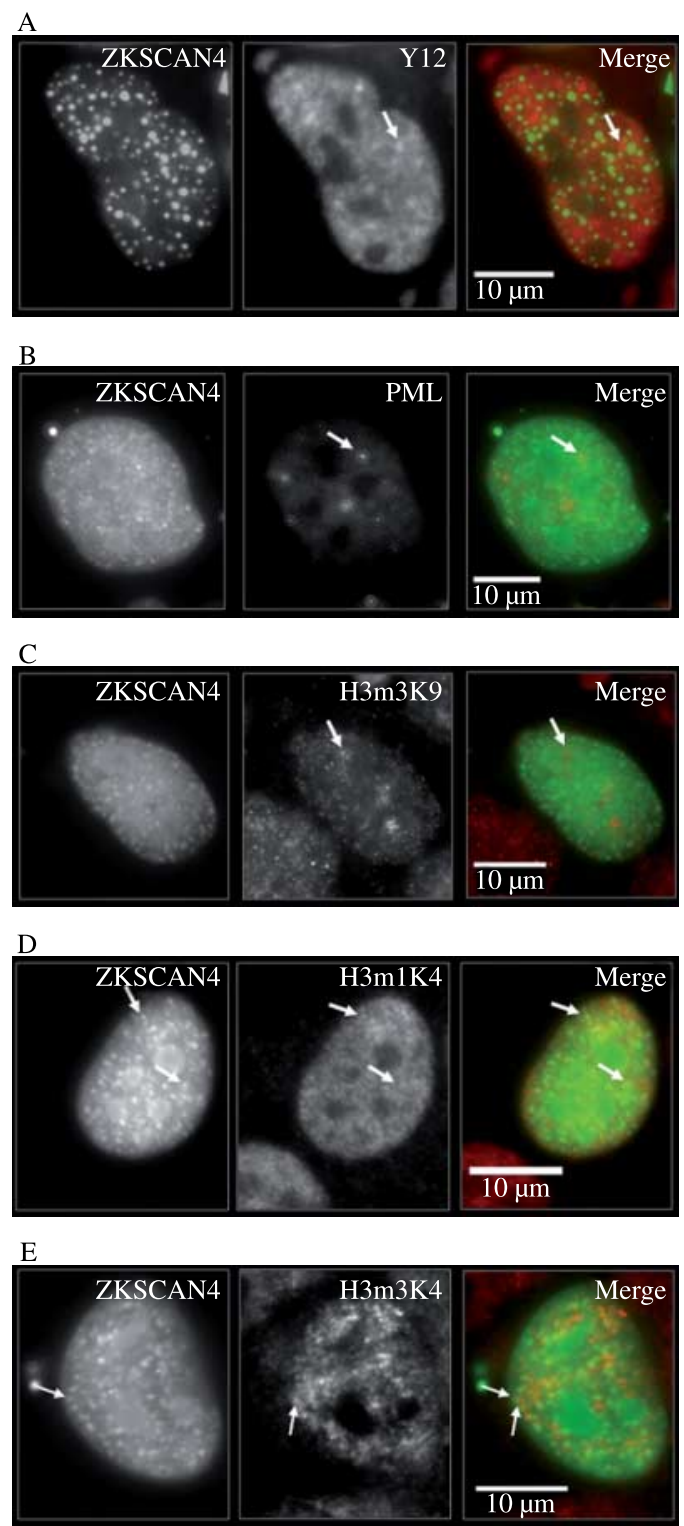

Figure 7 Localization of CFP-ZKSCAN4 in relation to wellcharacterized subnuclear structures. U2OS cells were transiently transfected with expression vectors encoding CFP-ZKSCAN4, fixated and stained with (A) Y12-antibody or (B) antibodies against PML, (C) histone H3-trimethyl-K9, (D) histone H3-monomethyl$\mathrm{K} 4$, or (E) histone $\mathrm{H} 3$-trimethyl-K4.

\section{Chromatin-dependent inhibition of GR-mediated transactivation}

A considerable number of proteins that are structurally similar to ZKSCAN4 exist in the human genome, and members of this group have been implicated in splicing and transcriptional regulation (Urrutia 2003). As ZKSCAN4 did not co-localize with Y12, a role in splicing seemed improbable. Another established role for similar proteins, which was also recently claimed for
ZKSCAN4, is in transcriptional repression by several mechanisms (Huang et al. 1999, Hennemann et al. 2003, $\mathrm{Li}$ et al. 2007). To assess a potential effect on glucocorticoid-dependent transcription, we tested the effect of ZKSCAN4 on a luciferase reporter construct driven by the glucocorticoid-responsive MMTVpromoter in a transient transfection assay. Transiently co-transfecting COS-7 cells with ZKSCAN4 and GR-expression constructs, as well as the MMTV-luciferase reporter, we did not find any significant effect of ZKSCAN4 on promoter activity of this episomal vector in the presence or absence of glucocorticoids (data not shown). However, some aspects of MMTV regulation are chromatin dependent and can only be assessed when the MMTV-LTR is integrated into chromosomal DNA (Deroo \& Archer 2001). We therefore stably integrated a MMTV-promoter-driven reporter construct into a GR-overexpressing Jurkat subline (Helmberg et al. 1995). Treatment of this bulk culture with dex leads to the induction of VNP (Venus-NLS-PEST; Nagoshi et al. 2004), which is an exclusively nuclear derivative of YFP with a short half-life of $\sim 20 \mathrm{~min}$, to closely reflect time-dependent cellular mRNA levels. Dex-dependent activity of the chromosomally integrated MMTV-promoter can thus be monitored by FACS analysis (Fig. 8A). We then infected cells of this culture with a selectable lentiviral construct resulting in constitutive overexpression of ZKSCAN4. In cells overexpressing ZKSCAN4, induction of VNP by treatment with dex for $14 \mathrm{~h}$ was markedly reduced compared with control cells (Fig. 8B): relative fluorescence intensity of ZKSCAN4 overexpressing cells was 45 vs 133 in VNP-positive control cells (window M1 in Fig. 8B).

\section{Discussion}

In this study, we used the full-length human GR to identify novel interacting proteins by applying the yeast reverse rRRS. By screening $\sim 800.000$ independent colonies, we identified 21 potential candidates. One of these, HSPA5, had been shown previously to bind to the GR in vitro (Hutchison et al. 1996), demonstrating that the system is in fact able to identify binding partners. We chose the reverse rRRS for two reasons. First, it allowed us to use the full-length human GR as bait, which is not possible in conventional yeast two-hybrid approaches. Secondly, it allowed the somewhat unusual step of anchoring the GR in the membrane, keeping it cytoplasmic even in the presence of hormone. Our intention in doing so was to facilitate interactions with cytoplasmic proteins. While most of the identified interaction candidates were indeed cytoplasmic, a number of nuclear proteins were identified as well, raising the question how these proteins were able to make contact with the membrane-bound receptor. 

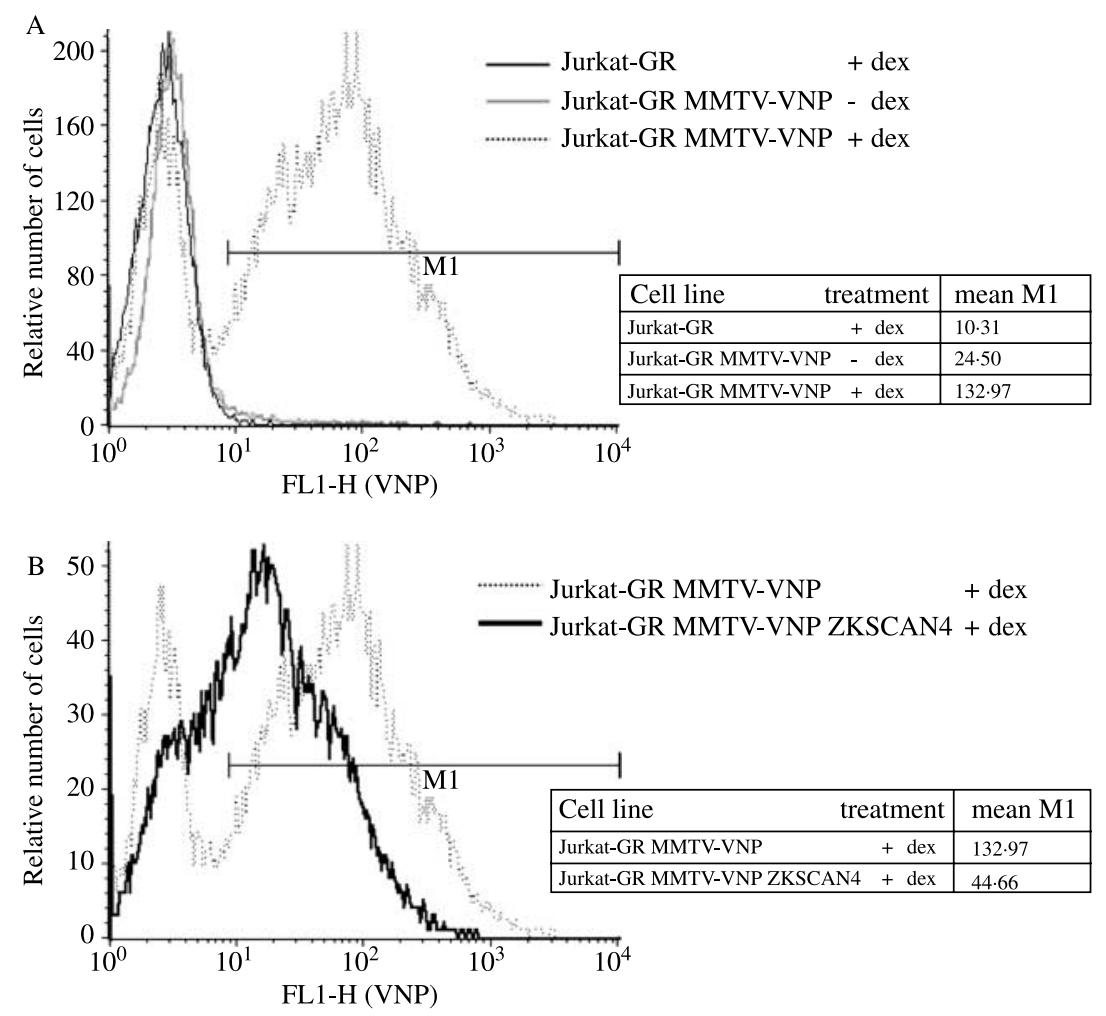

Figure 8 ZKSCAN4 dampens glucocorticoid responsiveness of a chromosomally integrated MMTV-promoter. (A) Dex induction of a chromosomally integrated MMTVVNP reporter. Jurkat-GR and Jurkat-GR MMTV-VNP cells were cultured for $14 \mathrm{~h}$ in the presence or absence of dex as indicated, and fluorescence intensity was measured by flow cytometry. (B) Effect of ZKSCAN4 on dex induction of the MMTV-VNP reporter. Jurkat-GR MMTV-VNP cells and Jurkat-GR MMTV-VNP ZKSCAN4 cells were cultured for $14 \mathrm{~h}$ in the presence of dex and fluorescence intensity was measured by flow cytometry. Both graphs represent overlays of curves showing relative fluorescence intensity against relative number of cells.

The simplest explanation would be that overexpression in the yeast cell resulted in a certain amount of the protein being cytoplasmic. We expected the presence of a glucocorticoid to be essential during the screening procedure. This proved not to be the case. Although much of the screening was done in the presence of deoxycorticosterone, which, in contrast to dex, was reported to be able to pass the yeast cell wall (Garabedian \& Yamamoto 1992), we did not identify any candidate demonstrating strictly ligand-dependent interaction. Positive clones remained positive, even in the absence of deoxycorticosterone. This was also true for nuclear proteins. Why did not more of the many known GR-interactors turn up in our screen? One limitation is probably the nature of our cDNA library, which was oligo-dT primed. The farther away the encoded interaction domain is from the poly-A tail, the less its chance to be represented in the library. Small interacting proteins or protein parts containing C-terminal interaction domains are far more likely to be identified than larger proteins with $\mathrm{N}$-terminal interaction domains. In addition, abundantly expressed yeast proteins may be sometimes crowding out competing similar human candidate proteins.

Of the proteins listed in Table 1, several are quite hydrophobic and belong to classes of proteins that have been found repeatedly in conventional yeast two-hybrid screens, e.g., ribosomal proteins and elongation factors. Although this does not exclude the possibility that they are GR interactors, a majority of them is likely to have been isolated due to unspecific interactions.

Of all assessed candidate proteins, interaction with the GR in human cell lines was most consistently confirmed for ZKSCAN4. As interaction was found in both yeast and human cell lines, it is likely to be direct, although it remains possible that interaction occurs via a third protein or a protein complex, as long as an analogous protein or complex is present in human as well as in yeast cells. According to the NCBI-database of expressed sequence tags (ESTs, June 2008), ZKSCAN4 is moderately expressed in many tissues, including, in descending order, cervix (82 transcripts per million), 
blood (64), tonsils (58), testis (51) and trachea (38), kidney (37), adrenal gland (30), mouth (29), skin (28), lung, (23), brain (19), spleen (18), uterus (17) and absent, e.g., in liver, intestine, and muscle.

While ZKSCAN4 was isolated as an interaction partner of the GR, it conceivably might also interact with other members of the nuclear receptor superfamily. To address this question, we specifically tested the PR, which, in contrast to GR, was not co-precipitated with ZKSCAN4. Obviously, this does not prove specificity for the GR, and further members of the superfamily will have to be tested individually.

ZKSCAN4 is a member of a large group of human zinc finger proteins that contain a KRAB- and a SCANbox. Some of them are thought to bind to DNA via their zinc finger domain, followed by transcriptional repression via the KRAB-box. The SCAN-box is thought to mediate homo- and hetero-dimerization with other SCAN-containing proteins (Williams et al. 1999). While most of these proteins have not been functionally characterized so far, individual members of this group have been reported to have roles in binding and splicing of RNA (Grondin et al. 1996), or to repress transcription from RNA polymerase I-, II- and IIIpromoters (reviewed by Urrutia 2003).

While co-localization of ZKSCAN4 with Y12, which decorates regions enriched in splicing factors would have suggested a role in splicing, no such association was found. The only potential, and very vague, hint on function from our co-localization experiments was an overlap in localization of ZKSCAN4 with nuclear regions showing histone $\mathrm{H} 3$ mono-methylated on lysine 4 , which might suggest an involvement in chromatin regulation (Sims et al. 2003, Schneider et al. 2004).

To assess an influence of ZKSCAN4 on the induction of the MMTV-promoter, which contains two glucocorticoid response elements, we selected two approaches. In a classical transient transfection assay with a naked-DNA reporter plasmid, we did not find any direct effect of ZKSCAN4 on glucocorticoid induction. However, if a potential repressive effect of ZKSCAN4 were to rely on a chromatin-condensing function, this cannot be detected by the transient transfection of an episomal reporter plasmid, but requires integration of the MMTV-reporter construct into a chromosome (Deroo \& Archer 2001). We therefore modified a GR-overexpressing Jurkat subline (Helmberg et al. 1995), adding a chromosomally integrated MMTV-driven VNP-reporter. In this setting, VNP fluorescence indicating MMTVpromoter activity was markedly reduced in the cells overexpressing ZKSCAN4. The difference in ZKSCAN4's ability to affect the identical promoter, dependent on the promoter's naked versus chromatinembedded state, argues for a chromatin-dependent repressive effect of ZKSCAN4. Whether this effect is specifically directed to glucocorticoid responsive genes or of a more general nature remains to be investigated.

\section{Declaration of interest}

The authors declare that there is no conflict of interest that could be perceived as prejudicing the impartiality of the research reported.

\section{Funding}

This work was supported by the Austrian Research Fund (P16013 to A Helmberg and P18571 to Reinhard Kofler and C Ploner) as well as by grants to K Ecker and A Helmberg from the Tyrolean Science Fund and the Austrian Cancer Society Tirol.

\section{Acknowledgements}

We thank Dr Ami Aronheim for the cdc25-2 yeast strain and related plasmids, Cornelia Wandke, Reinhard Sigl, Reinhard Kofler, Wolfgang Doppler, and Alexandra Lusser for their helpful discussions and Elisabeth Sparber and Veronika Rauch for expert technical assistance.

\section{References}

Aronheim A 2001 Ras signaling pathway for analysis of proteinprotein interactions. Methods in Enzymology 332 260-270.

Aronheim A, Engelberg D, Li N, al-Alawi N, Schlessinger J \& Karin M 1994 Membrane targeting of the nucleotide exchange factor Sos is sufficient for activating the Ras signaling pathway. Cell 78 949-961.

Broder YC, Katz S \& Aronheim A 1998 The ras recruitment system, a novel approach to the study of protein-protein interactions. Current Biology 8 1121-1124.

Colicelli J, Birchmeier C, Michaeli T, O’Neill K, Riggs M \& Wigler M 1989 Isolation and characterization of a mammalian gene encoding a high-affinity cAMP phosphodiesterase. PNAS 86 3599-3603.

Copik AJ, Webb MS, Miller AL, Wang Y, Kumar R \& Thompson EB 2006 Activation function 1 of glucocorticoid receptor binds TATA-binding protein in vitro and in vivo. Molecular Endocrinology 20 1218-1230.

Demaison C, Parsley K, Brouns G, Scherr M, Battmer K, Kinnon C, Grez M \& Thrasher AJ 2002 High-level transduction and gene expression in hematopoietic repopulating cells using a human immunodeficiency (correction of imunodeficiency) virus type 1-based lentiviral vector containing an internal spleen focus forming virus promoter. Human Gene Therapy 13 803-813.

Deroo BJ \& Archer TK 2001 Glucocorticoid receptor-mediated chromatin remodeling in vivo. Oncogene 20 3039-3046.

Dittmar KD, Demady DR, Stancato LF, Krishna P \& Pratt WB 1997 Folding of the glucocorticoid receptor by the heat shock protein (hsp) 90-based chaperone machinery. The role of p23 is to stabilize receptor.hsp90 heterocomplexes formed by hsp90.p60.hsp70. Journal of Biological Chemistry 272 21213-21220.

Doppler W, Windegger M, Soratroi C, Tomasi J, Lechner J, Rusconi S, Cato AC, Almlof T, Liden J, Okret S et al. 2001 Expression leveldependent contribution of glucocorticoid receptor domains for functional interaction with STAT5. Molecular and Cellular Biology 21 3266-3279.

Fields S \& Song O 1989 A novel genetic system to detect proteinprotein interactions. Nature 340 245-246.

Garabedian MJ \& Yamamoto KR 1992 Genetic dissection of the signaling domain of a mammalian steroid receptor in yeast. Molecular Biology of the Cell 3 1245-1257. 
Gottlicher M, Heck S, Doucas V, Wade E, Kullmann M, Cato AC, Evans RM \& Herrlich P 1996 Interaction of the Ubc9 human homologue with c-Jun and with the glucocorticoid receptor. Steroids 61 257-262.

Grondin B, Bazinet M \& Aubry M 1996 The KRAB zinc finger gene ZNF74 encodes an RNA-binding protein tightly associated with the nuclear matrix. Journal of Biological Chemistry 271 15458-15467.

Hedman E, Widen C, Asadi A, Dinnetz I, Schroder WP, Gustafsson JA \& Wikstrom AC 2006 Proteomic identification of glucocorticoid receptor interacting proteins. Proteomics 6 3114-3126.

Helmberg A, Auphan N, Caelles C \& Karin M 1995 Glucocorticoidinduced apoptosis of human leukemic cells is caused by the repressive function of the glucocorticoid receptor. EMBO Journal 14 452-460.

Hennemann H, Vassen L, Geisen C, Eilers M \& Moroy T 2003 Identification of a novel Kruppel-associated box domain protein, Krim-1, that interacts with c-Myc and inhibits its oncogenic activity. Journal of Biological Chemistry 278 28799-28811.

Hittelman AB, Burakov D, Iniguez-Lluhi JA, Freedman LP \& Garabedian MJ 1999 Differential regulation of glucocorticoid receptor transcriptional activation via AF-1-associated proteins. EMBO Journal 18 5380-5388.

Huang Z, Philippin B, O’Leary E, Bonventre JV, Kriz W \& Witzgall R 1999 Expression of the transcriptional repressor protein Kid-1 leads to the disintegration of the nucleolus. Journal of Biological Chemistry 274 7640-7648.

Hubsman M, Yudkovsky G \& Aronheim A 2001 A novel approach for the identification of protein-protein interaction with integral membrane proteins. Nucleic Acids Research 29 E18.

Hutchison KA, Dittmar KD, Stancato LF \& Pratt WB 1996 Ability of various members of the hsp70 family of chaperones to promote assembly of the glucocorticoid receptor into a functional heterocomplex with hsp90. Journal of Steroid Biochemistry and Molecular Biology 58 251-258.

Lamond AI \& Spector DL 2003 Nuclear speckles: a model for nuclear organelles. Nature Reviews. Molecular Cell Biology 4 605-612.

Lee EC, Yu D, Martinez de Velasco J, Tessarollo L, Swing DA, Court DL, Jenkins NA \& Copeland NG 2001 A highly efficient Escherichia colibased chromosome engineering system adapted for recombinogenic targeting and subcloning of BAC DNA. Genomics 73 56-65.

Li J, Wang Y, Fan X, Mo X, Wang Z, Li Y, Yin Z, Deng Y, Luo N, Zhu C et al. 2007 ZNF307, a novel zinc finger gene suppresses $\mathrm{p} 53$ and p21 pathway. Biochemical and Biophysical Research Communications 363 895-900.

Mumberg D, Muller R \& Funk M 1994 Regulatable promoters of Saccharomyces cerevisiae: comparison of transcriptional activity and their use for heterologous expression. Nucleic Acids Research 22 $5767-5768$.

Nagoshi E, Saini C, Bauer C, Laroche T, Naef F \& Schibler U 2004 Circadian gene expression in individual fibroblasts: cell-autonomous and self-sustained oscillators pass time to daughter cells. Cell 119 693-705.

Necela BM \& Cidlowski JA 2004 Mechanisms of glucocorticoid receptor action in noninflammatory and inflammatory cells. Proceedings of the American Thoracic Society 1 239-246.

Petitjean A, Hilger F \& Tatchell K 1990 Comparison of thermosensitive alleles of the CDC25 gene involved in the cAMP metabolism of Saccharomyces cerevisiae. Genetics 124 797-806.

Ploner C, Rainer J, Niederegger H, Eduardoff M, Villunger A, Geley S \& Kofler R 2008 The BCL2 rheostat in glucocorticoid-induced apoptosis of acute lymphoblastic leukemia. Leukemia 22 370-377.
Pratt WB \& Toft DO 1997 Steroid receptor interactions with heat shock protein and immunophilin chaperones. Endocrine Reviews 18 306-360.

Pratt WB, Morishima Y, Murphy M \& Harrell M 2006 Chaperoning of glucocorticoid receptors. Handbook of Experimental Pharmacology 172 111-138.

Presul E, Schmidt S, Kofler R \& Helmberg A 2007 Identification, tissue expression, and glucocorticoid responsiveness of alternative first exons of the human glucocorticoid receptor. Journal of Molecular Endocrinology 38 79-90.

Riml S, Schmidt S, Ausserlechner MJ, Geley S \& Kofler R 2004 Glucocorticoid receptor heterozygosity combined with lack of receptor auto-induction causes glucocorticoid resistance in Jurkat acute lymphoblastic leukemia cells. Cell Death and Differentiation 11 S65-S72.

Sapolsky RM, Romero LM \& Munck AU 2000 How do glucocorticoids influence stress responses? Integrating permissive, suppressive, stimulatory, and preparative actions Endocrine Reviews 21 55-89.

Schimmer B \& Parker K 2005 Adrenocortical steroids. In Goodman and Gilman's The Pharmacological Basis of Therapeutics, 11 edn, pp 1593-1610. Eds L Brunton, J Lazo \& K Parker. New York: McGraw-Hill.

Schneider R, Bannister AJ, Myers FA, Thorne AW, Crane-Robinson C \& Kouzarides T 2004 Histone H3 lysine 4 methylation patterns in higher eukaryotic genes. Nature Cell Biology 6 73-77.

Sengupta S \& Wasylyk B 2001 Ligand-dependent interaction of the glucocorticoid receptor with p53 enhances their degradation by Hdm2. Genes and Development 15 2367-2380.

Sengupta S, Vonesch JL, Waltzinger C, Zheng H \& Wasylyk B 2000 Negative cross-talk between p53 and the glucocorticoid receptor and its role in neuroblastoma cells. EMBO Journal 19 6051-6064.

Sims RJ III, Nishioka K \& Reinberg D 2003 Histone lysine methylation: a signature for chromatin function. Trends in Genetics 19 629-639.

Stellato C 2004 Post-transcriptional and nongenomic effects of glucocorticoids. Proceedings of the American Thoracic Society 1 255-263.

Urrutia R 2003 KRAB-containing zinc-finger repressor proteins. Genome Biology 4231.

Williams AJ, Blacklow SC \& Collins T 1999 The zinc finger-associated SCAN box is a conserved oligomerization domain. Molecular and Cellular Biology 19 8526-8535.

Yang L, Guerrero J, Hong H, DeFranco DB \& Stallcup MR 2000 Interaction of the tau2 transcriptional activation domain of glucocorticoid receptor with a novel steroid receptor coactivator, Hic-5, which localizes to both focal adhesions and the nuclear matrix. Molecular Biology of the Cell 11 2007-2018.

Yu D, Ellis HM, Lee EC, Jenkins NA, Copeland NG \& Court DL 2000 An efficient recombination system for chromosome engineering in Escherichia coli. PNAS 97 5978-5983.

Yudt MR \& Cidlowski JA 2001 Molecular identification and characterization of a and $\mathrm{b}$ forms of the glucocorticoid receptor. Molecular Endocrinology 15 1093-1103.

Zhong S, Salomoni P \& Pandolfi PP 2000 The transcriptional role of PML and the nuclear body. Nature Cell Biology 2 E85-E90.

Received in final form 21 October 2008

Accepted 13 November 2008

Made available online as an Accepted Preprint 13 November 2008 